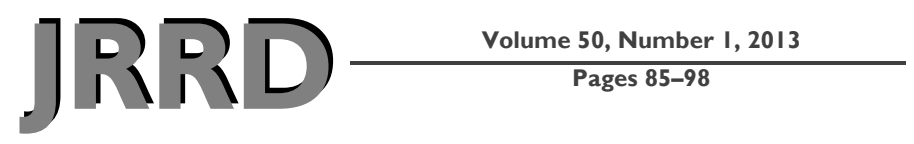

\title{
Interaction of poststroke voluntary effort and functional neuromuscular electrical stimulation
}

\author{
Nathaniel Makowski; ${ }^{*}$ Jayme Knutson, PhD; ${ }^{1-2}$ John Chae, MD; ${ }^{1-2}$ Patrick Crago, PhD ${ }^{\mathbf{1}}$ \\ ${ }^{1}$ Department of Biomedical Engineering, Case Western Reserve University, Cleveland, OH; and Cleveland Functional \\ Electrical Stimulation Center, Cleveland, $\mathrm{OH} ;{ }^{2}$ Department of Physical Medicine and Rehabilitation, MetroHealth \\ Medical Center, Cleveland, $\mathrm{OH}$
}

\begin{abstract}
Functional electrical stimulation (FES) may be able to augment functional arm and hand movement after stroke. Poststroke neuroprostheses that incorporate voluntary effort and FES to produce the desired movement must consider how forces generated by voluntary effort and FES combine, even in the same muscle, in order to provide an appropriate level of stimulation to elicit the desired assistive force. The goal of this study was to determine whether the force produced by voluntary effort and FES add together independently of effort or whether the increment in force depends on the level of voluntary effort. Isometric force matching tasks were performed under different combinations of voluntary effort and FES. Participants reached a steady level of force, and while attempting to maintain a constant effort level, FES was applied to augment the force. Results indicate that the increment in force produced by FES decreases as the level of initial voluntary effort increases. Potential mechanisms causing the change in force output are proposed, but the relative contribution of each mechanism is unknown.
\end{abstract}

Key words: force, functional electrical stimulation, isometric, motor control, motor unit overlap, neuroprosthesis, reach, recruitment, stroke, triceps, upper limb.

\section{INTRODUCTION}

Stroke is a leading cause of disability in the United States. Six months poststroke, 50 percent of people with ischemic stroke over 64 years old still have a degree of upper-limb hemiparesis that limits arm and hand function
[1], making bimanual tasks difficult, if not impossible. Hemiparesis is worsened by disuse and cocontraction patterns across multiple joints (i.e., synergy patterns) [2]. These synergy patterns have been well quantified [3-4] and appear to be expressed in proportion to effort [5-6]. Greater effort to abduct the arm increases the involuntary flexor contractions that oppose the desired movement.

Functional electrical stimulation (FES) of paretic muscles can potentially elicit functional limb movements [7], such as reaching and hand opening [7-9]. For example, electrical stimulation of finger extensors in a person with stroke [10-14] can produce hand opening while he or she is relaxed. However, when exerting effort to open the hand during stimulation, the hand does not open as much as when the user remains relaxed, presumably because the effort to open the hand produces involuntary finger flexor contractions [10,15-16]. Therefore, in order to receive maximum hand opening from the stimulation, the user must remain relaxed, which is unnatural and runs counter to motor rehabilitation principles that encourage

\footnotetext{
Abbreviations: $\mathrm{ANOVA}=$ analysis of variance, $\mathrm{DOF}=$ degree of freedom, EMG = electromyogram, FES = functional electrical stimulation, $\mathrm{vFmax}=$ maximum voluntary force.

*Address all correspondence to Nathaniel Makowski; Department of Biomedical Engineering, Case Western Reserve University, 2071 Martin Luther King Jr. Dr, Cleveland, OH 44106. Email: nhm6@cwru.edu http://dx.doi.org/10.1682/JRRD.2011.04.0068
} 
active attempts to produce functional movement. Our long-term objective is to develop an upper-limb FES system that a person with stroke controls with residual electromyogram (EMG) signals recorded from the affected upper limb. Thus, the control strategy will require the user to exert some effort to produce desired arm and hand movements.

In order to develop an effective neuroprosthesis that integrates both stimulation and voluntary effort in an efficient and natural way, we must understand their interaction. Previous studies have reported that exerting effort reduces the effectiveness of stimulation both across joints [9] and within the same joint [17]. As a result of changes to central commands and reflexes after stroke, differences could exist between how volitional and electrically stimulated forces interact in participants with stroke and nondisabled participants. The relationship between the level of voluntary effort and the effect of FES in the same direction in stroke has not been quantified. It may be possible that small or moderate amounts of effort do not completely limit the effects of stimulation.

The goal of this study was to determine how voluntary effort and electrical stimulation of elbow extensors combine to produce force after stroke (i.e., do the two forces add linearly?). To quantify the relationship between voluntary effort and the effect of FES, we stimulated the elbow extensor muscle (triceps) of participants with stroke and measured the isometric force produced when participants exerted various levels of simultaneous voluntary effort to push forward, extending the elbow. There are two hypotheses for this study: (1) electrical stimulation of elbow extensors produces greater forward force than voluntary effort alone, even in the presence of simultaneous voluntary effort, and (2) as the level of vol- untary effort increases, the amount of force added by stimulation decreases.

\section{METHODS}

\section{Participants}

We enrolled six participants who had experienced a single stroke (Table 1) and were recruited from an outpatient stroke clinic. The primary inclusion criteria included (1) being at least 6 mo poststroke, (2) the ability to reach at least 20 percent of full passive reach starting from the closest the hand can passively sit in front of the shoulder while an investigator manually supported the elbow and wrist at $90^{\circ}$ of shoulder abduction, and (3) the ability to follow three-stage commands. Exclusion criteria included (1) shoulder pain; (2) uncompensated spatial neglect; (3) apraxia; (4) insensate chest, arm, or forearm; and (5) impaired cognition or communication. We excluded one participant from data analysis because of his great difficulty completing the tasks involved in this study.

\section{Setup}

Participants performed a series of isometric upperlimb forward force generation tasks (described later) while seated with their arm in two different standardized positions (near or far). Each participant sat in front of a computer monitor with his or her trunk restrained (Figure 1). Figure 1(a) shows the reference frame used for force directions.

The arm was positioned at $90^{\circ}$ shoulder abduction with the shoulder flexed and elbow extended to bring the wrist directly anterior to the shoulder at a distance from the shoulder of either 50 (near) or 75 (far) percent of the maximum passive reach. We compared different positions

Table 1.

Participant demographics.

\begin{tabular}{ccccccc}
\hline Participant & Age (yr) & Sex & Affected Side & Dominant Side & $\begin{array}{c}\text { Time Since } \\
\text { Stroke (mo) }\end{array}$ & $\begin{array}{c}\text { FMA Score } \\
\text { (arm) }\end{array}$ \\
\hline 1 & 61 & $\mathrm{M}$ & $\mathrm{R}$ & $\mathrm{R}$ & 22 & $17(16)$ \\
2 & 53 & $\mathrm{M}$ & $\mathrm{L}$ & $\mathrm{R}$ & 11 & $48(26)$ \\
3 & 43 & $\mathrm{~F}$ & $\mathrm{R}$ & $\mathrm{R}$ & 10 & $26(21)$ \\
4 & 57 & $\mathrm{M}$ & $\mathrm{R}$ & $\mathrm{R}$ & 24 & $37(23)$ \\
5 & 79 & $\mathrm{M}$ & $\mathrm{R}$ & $\mathrm{R}$ & 25 & $25(20)$ \\
$6^{\dagger}$ & 48 & $\mathrm{M}$ & $\mathrm{R}$ & $\mathrm{R}$ & 56 & $18(16)$ \\
\hline
\end{tabular}

${ }^{\text {*}}$ Shown as total FMA score (66 points maximum), with shoulder/elbow/forearm score in parentheses (34 points maximum).

${ }^{\dagger}$ Excluded from data analysis.

$\mathrm{F}=$ female, FMA = Fugl-Meyer Motor Assessment, $\mathrm{L}=$ left, $\mathrm{M}=$ male, $\mathrm{R}=$ right. 
(a)

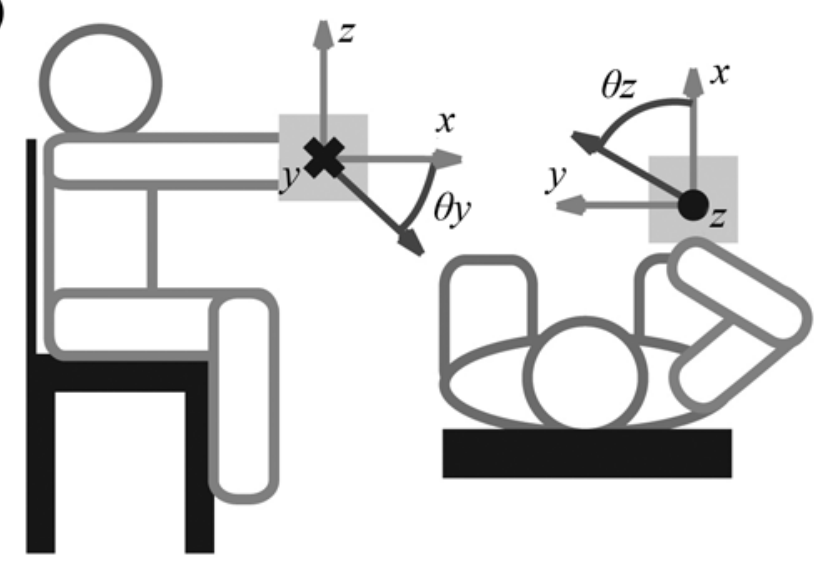

(b)

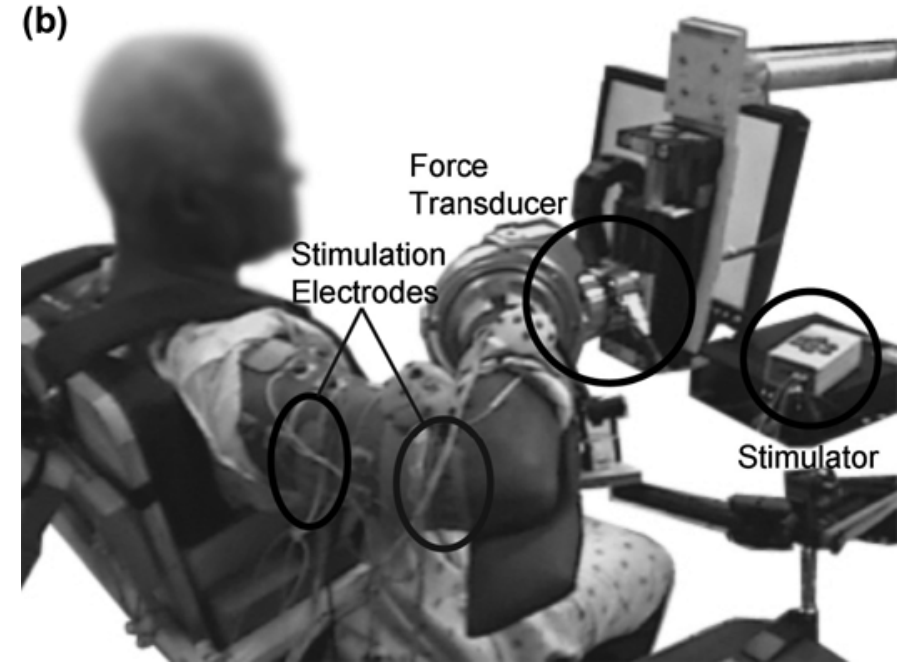

Figure 1.

(a) General setup of participant's arm orientation: $x-, y-$, and $z-$ axes show coordinate frame for measurements and $\theta y$ and $\theta z$ indicate directions of rotations for hypothetical target placement, as indicated by darker arrows. (b) Setup showing near arm location and relative equipment locations.

to determine whether arm posture affected the interaction. A fiberglass cast over the wrist and forearm connected the wrist to a 2-degree-of-freedom (DOF) gimbal that was attached to a 6-DOF force transducer (model 30E15A-U560A, JR3 Inc; Woodland, California). The forearm was supported by an elevating mobile arm support (JAECO/Rancho MultiLink Arm with Elevation Assist, JAECO Orthopedic; Hot Springs, Arkansas) in addition to the gimbal in order to reduce arm-weight pressure at the proximal edge of the cast. Less than $0.059 \mathrm{~N}$ of horizontal force was required to move the arm support in the horizontal plane. For the participant with the smallest stimulated force, the force required to move the arm support was less than 0.9 percent of his or her stimulated force. The elevating mobile arm support used rubber bands to apply a passive vertical force. The rubber bands providing the support were highly compliant, and the stiffness of the device was less than $9 \mathrm{~N} / \mathrm{m}$. We measured the maximum vertical elbow movement during all of the trials to determine the maximum force transmitted through the support. The participant whose movement could have the largest effect on their normalized force had a maximum vertical elbow translation of $0.024 \mathrm{~m}$ during a single trial. The maximum active vertical force that could have been transmitted through the support during an entire trial was 3.1 percent of the stimulated force magnitude. The force transmitted through the support would have been less in the rest of the trials. While less than 3.1 percent of the stimulated force magnitude was transmitted through the support, the transducer recorded the majority of the active vertical forces. Isometric endpoint forces generated by pushing forces were low-pass filtered at $5 \mathrm{~Hz}$, sampled at $60 \mathrm{~Hz}$, and then down sampled to $12 \mathrm{~Hz}$.

We placed surface stimulation electrodes $(0.038 \times$ $0.089 \mathrm{~m}$ ) over the triceps to generate elbow extension torques. We placed the stimulation electrodes approximately over the long and lateral heads of the triceps. It is difficult to estimate the proportional contributions of the different heads of the triceps to the stimulated force because the primary target was simply the radial nerve. We delivered current pulses through a custom computercontrolled stimulator with a pulse frequency of $35 \mathrm{~Hz}$, pulse amplitude of 40 to $60 \mathrm{~mA}$, and pulse width that could be modulated from 0 to $255 \mu$ s. We set the pulse amplitude and pulse width for each participant to levels that produced the desired magnitude (discussed later) of isometric force without pain when he or she was relaxed with the upper arm fully supported. The stimulator ramped the pulse width from $0 \mu$ s to the preset level in $0.5 \mathrm{~s}$ corresponding to cues during the tasks and remained on for $3.0 \mathrm{~s}$.

We placed position tracking markers (light-emitting diode clusters) on the trunk, upper arm, and forearm to record limb movement relative to the trunk with an optical tracking system (Northern Digital Inc; Bakersfield, California) in order to confirm that the participant did not move during the trials. 


\section{Experimental Procedures}

Prior to any isometric force task sessions, an occupational therapist performed a Fugl-Meyer Motor Assessment [18] to characterize the degree of upper-limb motor impairment. Participants then returned to the laboratory for two to four sessions to learn the force generation tasks and become accustomed to the sensation of surface stimulation. During two final sessions, we collected force and kinematic data for analysis.

Participants performed upper-limb isometric forward force generation tasks that included various combinations of two factors: voluntary effort and FES. Before the participants began the force generation tasks, we demonstrated how to interpret force magnitude and direction feedback on a computer monitor; this was repeated at the beginning of every session. For each force generation task, we presented the participants with a target force direction and magnitude. The target direction was the direction closest to a line extending directly anterior from the shoulder in which the participant could consistently generate voluntary force. The target magnitude was a percentage of the maximum voluntary force (vFmax) that participants could maintain in the target direction. We verbally encouraged participants to maintain the target force magnitude in a direction as close to anterior as possible. During these tasks, the percentage of the end-point $v F m a x$ served as an estimate of effort level. We considered zero effort to be the participant relaxed and not actively exerting any force. We considered maximum effort $(100 \%)$ to be when he or she exerted vFmax. Measuring end-point force provided a quantifiable estimate of the participant's effort during the tasks.

Because of difficulty generating a completely anterior force, we chose a target direction for voluntary force for each participant in a direction he or she could consistently generate force. We measured the end-point vFmax that each participant could generate in the target direc- tion. We instructed the participant to reach a target force and try to maintain that force level. The computer monitor displayed both the force generated and target force. We determined the magnitude of vFmax by starting with a level that was initially well within the participant's achievable range. We then incrementally raised the magnitude of the target in successive trials until participants could no longer generate sufficient force to reach the target. We verbally encouraged participants to generate as much force as possible while we determined their vFmax. We defined the maximum magnitude force that participants could maintain in the target direction for $1.5 \mathrm{~s}$ as their maximum end-point vFmax, which we used to determine the target force magnitude for each participant.

During the tasks, tolerances on the target force allowed participants to stay within the target without maintaining an exact force. The vertical force tolerance in either direction was 5 percent of the force being generated in the target direction. The horizontal force tolerance from the target was 25 percent of the magnitude of the target force. Participants who could generate force more consistently had smaller tolerances on their targets. The reason for a 3-dimensional force matching task was to elicit consistent voluntary force levels by allowing proportional cocontraction levels. While it has been shown that people with stroke generate secondary forces in constrained patterns during maximum voluntary contractions [19], at submaximal effort levels people can generate movement and force outside of these patterns $[5,20]$.

Participants repeated the following force matching tasks during both the practice and data collection sessions. He or she repeated each trial type four to eight times per session depending on the participant. Table 2 shows each participant's average rotations for the isometric force target placement around the $y$ - and $z$-axes (Figure 1(a)), the range of repetitions of the number of trials,

Table 2.

Participants' average preferred directions for voluntary force generation, range of repetitions for various tasks, and average error during hold prior to removing feedback and adding stimulation.

\begin{tabular}{cccc}
\hline Participant & $\begin{array}{c}\text { Preferred } \\
\left.\text { Direction Rotations ( }{ }^{*}\right)^{*}\end{array}$ & Repetitions & Error (\%) \\
\hline 1 & $3 / 17$ & $4-5$ & 17 \\
2 & $14 / 25$ & $5-7$ & 19 \\
3 & $48 / 4$ & $6-7$ & 16 \\
4 & $8 / 14$ & $4-7$ & 16 \\
5 & $24 / 12$ & $5-8$ & 16 \\
${ }^{*} \theta z / \theta y$. & & \\
\hline \hline
\end{tabular}


and the total average error that participants had during the $1.5 \mathrm{~s}$ prior to removing feedback and adding stimulation.

\section{Voluntary Effort Maintenance Task}

Participants exerted effort to match a force magnitude that was 20 or 50 percent of their vFmax in the target force direction. Once participants reached the target magnitude and maintained a steady force for $1.5 \mathrm{~s}$, we removed visual force feedback while instructing them to continue exerting the same level of effort.

\section{Stimulation Force Task}

We stimulated each participant's triceps to generate a force magnitude equal to 20 percent of the anterior component of the participant's vFmax in the target direction. We instructed participants to remain relaxed and did not provide visual force feedback. A $0.5 \mathrm{~s}$ linear ramp increase in pulse width began when we turned the stimulation on. Stimulation stayed on for at least $3 \mathrm{~s}$.

\section{Combined Voluntary Effort and Stimulation Force Task}

These tasks started in a similar manner to the voluntary effort maintenance task, with participants generating either 20 or 50 percent of vFmax. When they had maintained a steady force for $1.5 \mathrm{~s}$, we removed visual force feedback and instructed them to "keep pushing in the same way you're pushing, even when the stimulation comes on" in order to have them maintain the same level of effort during stimulation, similar to the "do not intervene" instructions in Crago et al., Burgess et al., and Trumbower et al. [21-23]. We applied the same stimulation parameters used in the stimulation force task. Figure 2 shows an example of this combined effort and stimulation force trial.

\section{Data Analysis}

For each participant in each position, we calculated the change in force in the stimulated direction after feedback removal and/or stimulation onset. Separately, for each participant and position, we ensemble averaged the stimulation-alone trials where the participant remained relaxed from the time when the stimulation ramp was started to $2 \mathrm{~s}$ after that point, i.e., $1.5 \mathrm{~s}$ after the stimulation reached its plateau, in order to determine the change in force in the direction of the stimulation. We considered the maximum force magnitude generated during that $2 \mathrm{~s}$ window the maximum stimulated force. Then, using that stimulation-alone ensemble averaged data set, we found

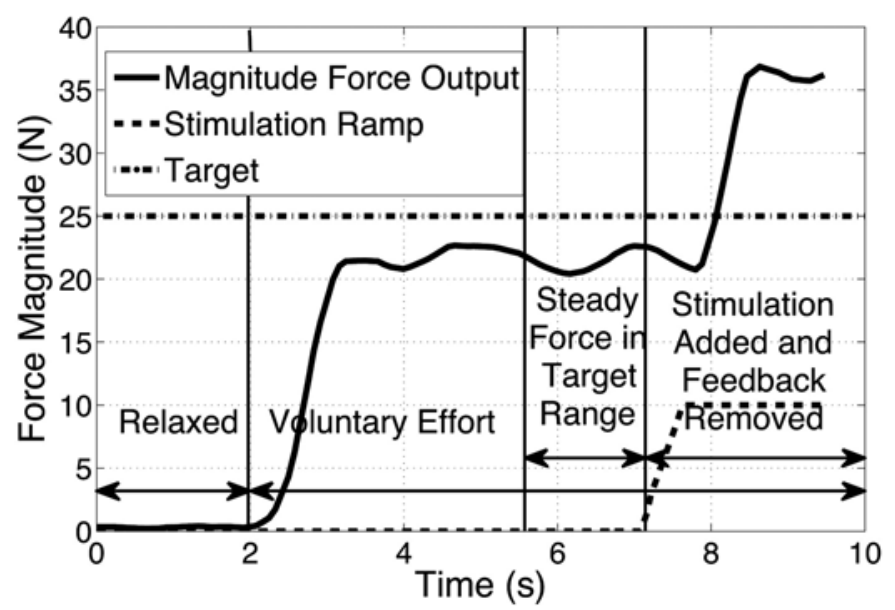

Figure 2.

Example of combined low voluntary effort and stimulation trial performed by participant 1.

two time points. The first time point was when the stimulation-alone force reached 5 percent and the second was when the stimulation-alone force reached 90 percent of the maximum stimulated force. Using these two time points, we calculated the change in force in the direction of the triceps stimulation alone with respect to when we removed feedback and turned on stimulation. We calculated the change in force for all three conditions as the difference between the forces at the points where the stimulated force was at 5 and 90 percent of its maximum stimulated force. To reduce variance in each trial from changes in voluntary effort while feedback was removed and the arm was being stimulated, we averaged together the force at 90 percent with the forces from 85 to 95 percent. To compare force changes caused by stimulation across participants and arm positions, we normalized the changes in force by the vFmax that each participant volitionally generated in the direction of stimulation at each arm position.

We compared the normalized force increments generated by the stimulation across different levels of initial voluntary effort to assess whether the dependence on initial voluntary effort was statistically significant $(p<$ 0.05 ). We used an analysis of variance (ANOVA) to compare the change in force using initial effort level (none, low, and high) and position (near and far) as factors while blocking for participant. If values were statistically significantly different, we repeated the analysis while including participants as a fixed factor. Then we used the 
Tukey-Kramer comparison of means to determine statistical difference between force increments for separate factors. To evaluate the effects of the change in force during the voluntary force maintenance task's effect on the combined task, we subtracted the average change in force during the voluntary force maintenance task using the time points described previously from each of the combined voluntary effort and stimulation force tasks. We repeated the analysis comparing the stimulation-alone values to the combined effort and stimulation values minus the average of the change during the voluntary force maintenance task.

\section{RESULTS}

At all three levels of voluntary effort, the addition of stimulation increased the force, as illustrated by the superimposed individual trials from one participant (Figure 3(a)). However, the force increment caused by stimulation decreased as the voluntary force (effort) increased, as shown by the superimposed incremental force responses in Figure 3(b).

To compare force changes caused by stimulation across participants and arm positions, we normalized the changes in force from stimulation by the component of the vFmax that is in the direction of stimulation for each participant and arm position. For all five participants at both arm positions, the combined stimulation and voluntary force increased with increasing voluntary force (effort) (Figure 4(a), (c)). However, the size of the force increment after the onset of stimulation decreased with increasing voluntary force (effort) in all cases except for one: participant 3 at the near position (Figure 4(b), (d)).

Using the previously described statistical model that blocks for participant and includes initial effort level and position as factors, we evaluated the response to stimulation. Main effects were effort level, position, and participant, while interaction effects included two-way combinations of all three main effects. All of the interaction effects were statistically significant (participant and effort: $p<0.01$, participant and position: $p<0.01$, effort and position: $p=0.03)$. The main effect of effort was significant $(p=0.01)$, while participant and position were not statistically significant $(p=0.19$ and $p=0.09$ ). The sum of squares from the different initial effort levels is 0.41 , while the sum of squares for the interaction of participant and initial effort is $0.11 . R^{2}$ was 87.3 percent. (a)

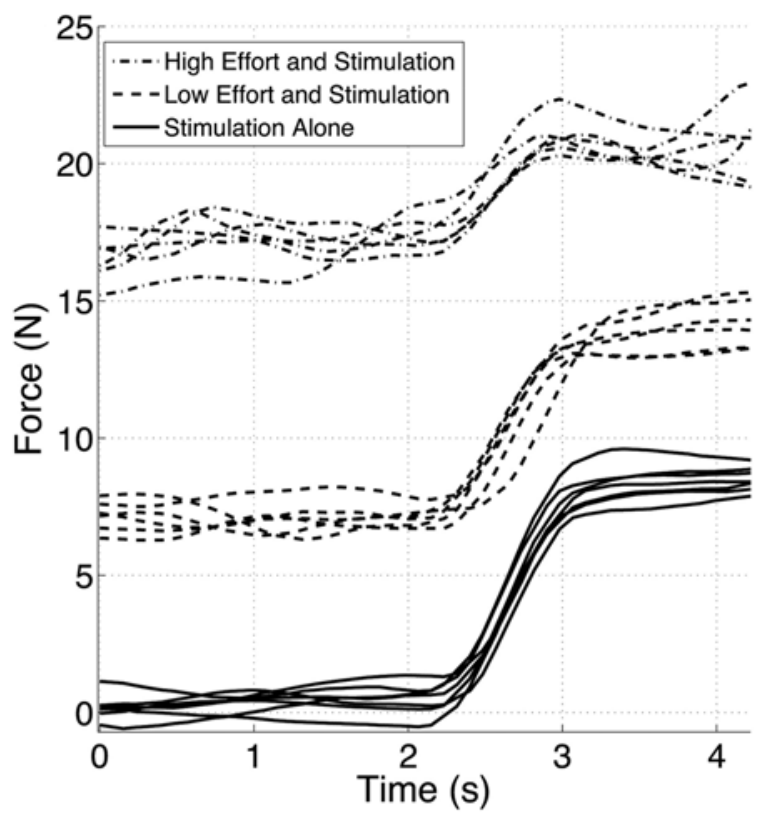

(b)

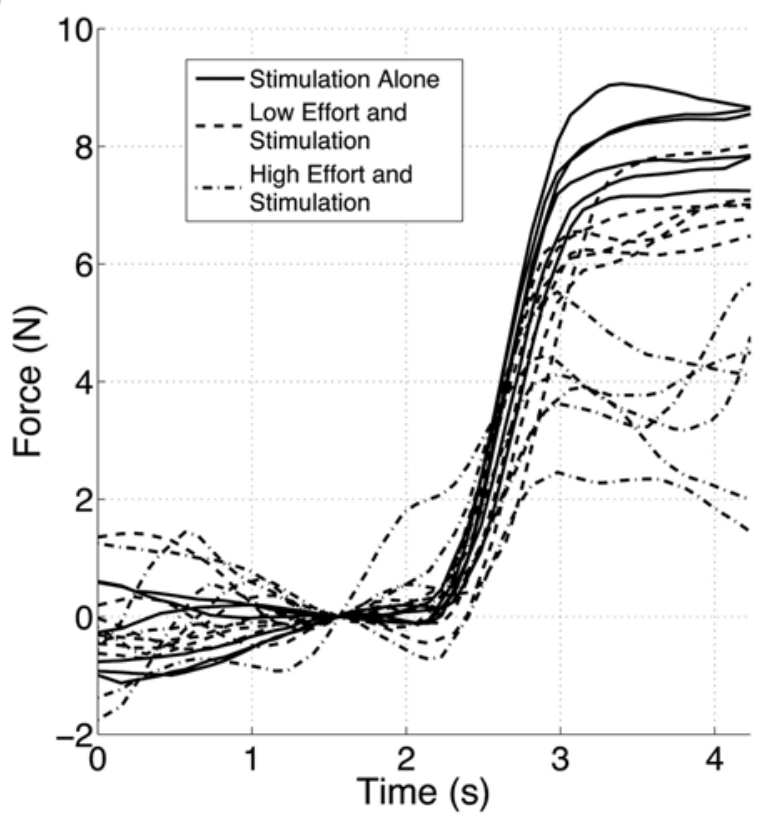

Figure 3.

Examples from participant 5 of stimulation response while he maintains different levels of voluntary force (effort). (a) Total force in stimulated direction before and after onset of stimulation. (b) Change in force from time point when feedback was removed and stimulation ramp was started. Solid lines are trials where participant is relaxed and exerting no effort. Dotted lines represent low (20\%) voluntary effort during onset of stimulation. Dashed lines represent moderate (50\%) voluntary effort during same stimulation. 
(a)

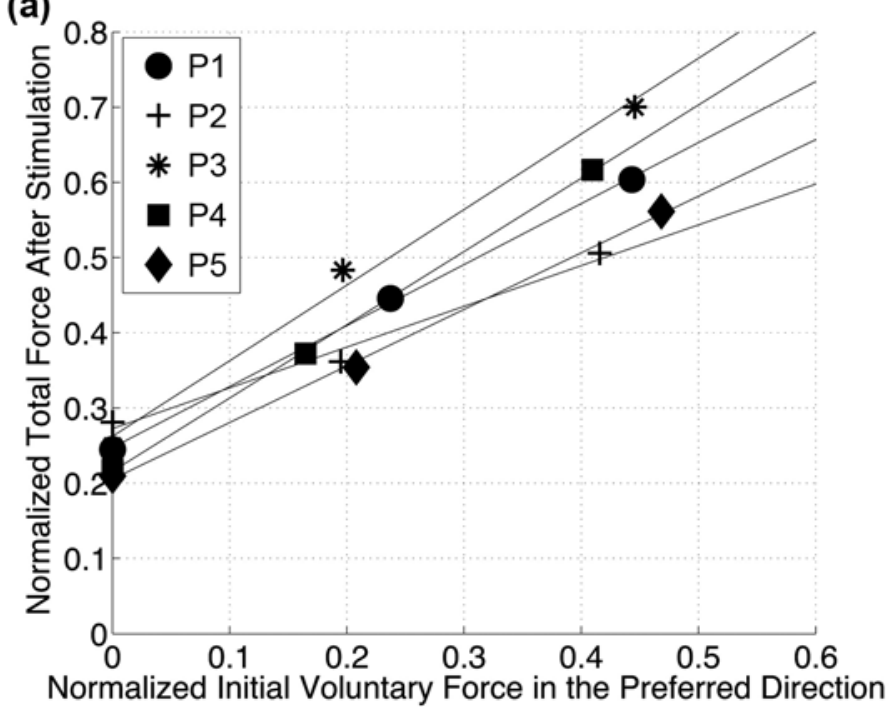

(c)

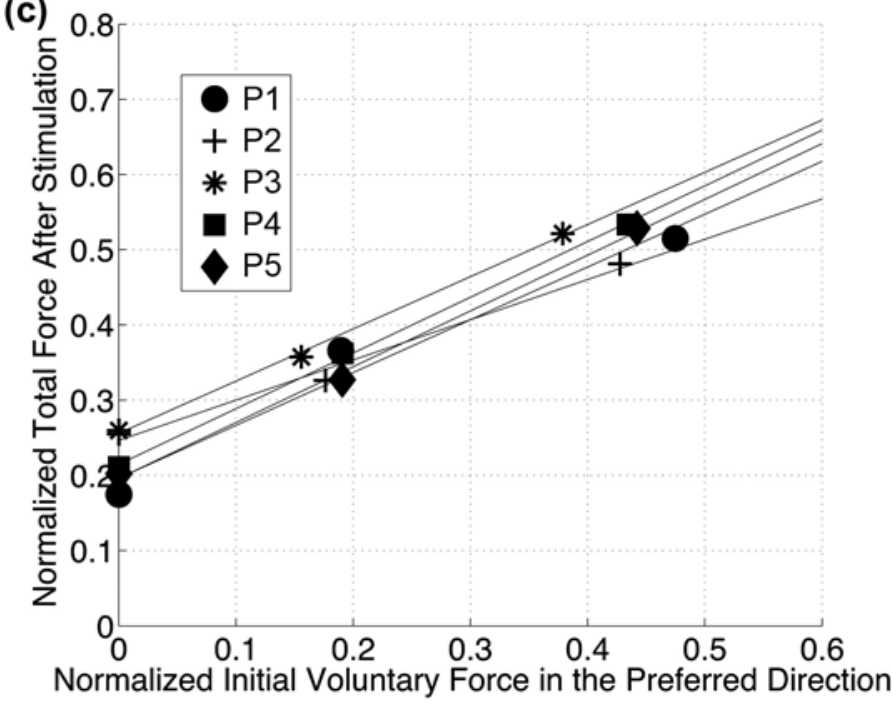

(b)

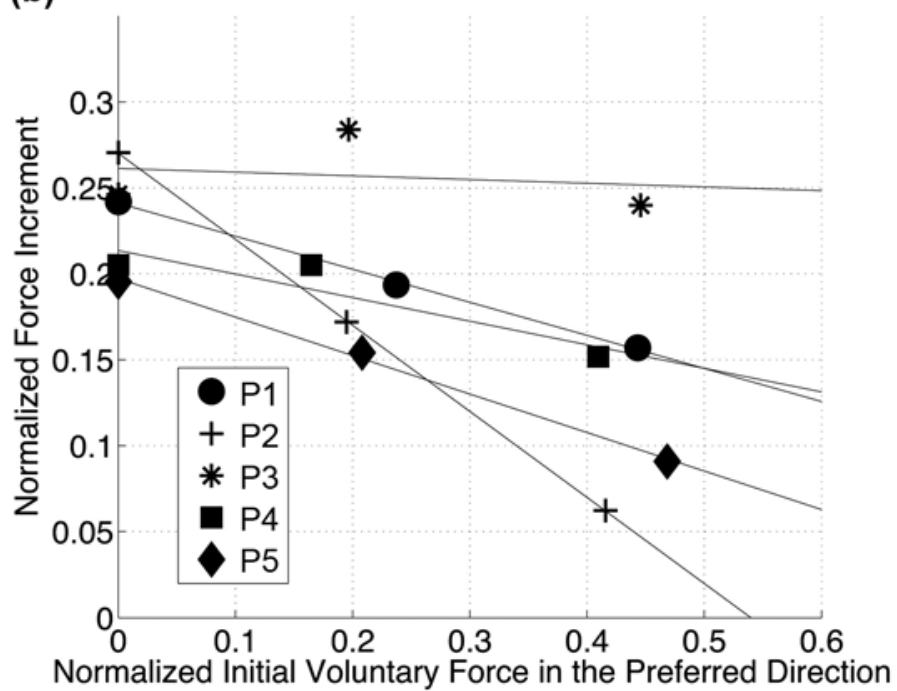

(d)

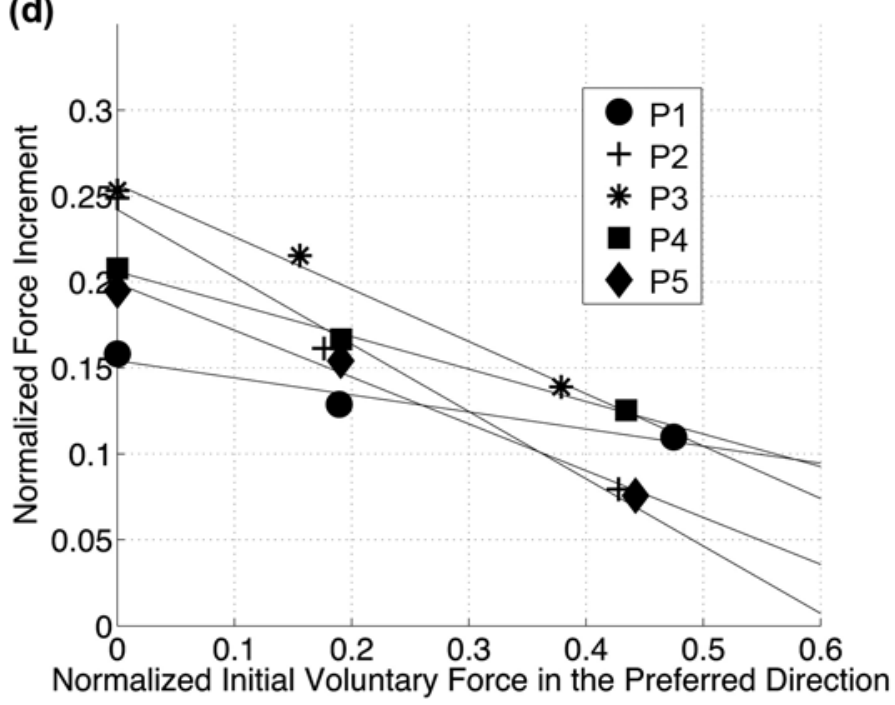

Figure 4.

Total force after onset of stimulation normalized by magnitude of maximum voluntary force in (a) near and (c) far position. Normalized force increment after onset of stimulation in (b) near and (d) far position. Horizontal axis represents normalized voluntary force prior to onset of stimulation. Vertical axis shows normalized force response. Lines are fitted to force responses showing change in force as function of normalized voluntary force. $\mathrm{P}=$ participant.

Figure 5 shows data averaged across participants. Despite changes in $p$-values, the same effects were significant when we subtracted the change in force during the voluntary effort maintenance task from the combined force increment. Post hoc analyses provided more insight into which positions and effort levels were statistically significant for individual participants $(p<0.05)$. In the near position, participant 3 was not statistically signifi- cantly different from stimulation alone at low or high effort and participant 4 was not significantly different at low effort (Figure 4(b)). Participants 2 and 5 were statistically different in all positions and at all effort levels. Participant 1 was not statistically significantly different in the far position at low or high effort; however, the data exhibited a similar downward trend and an ANOVA applied to that data set revealed significant differences at 


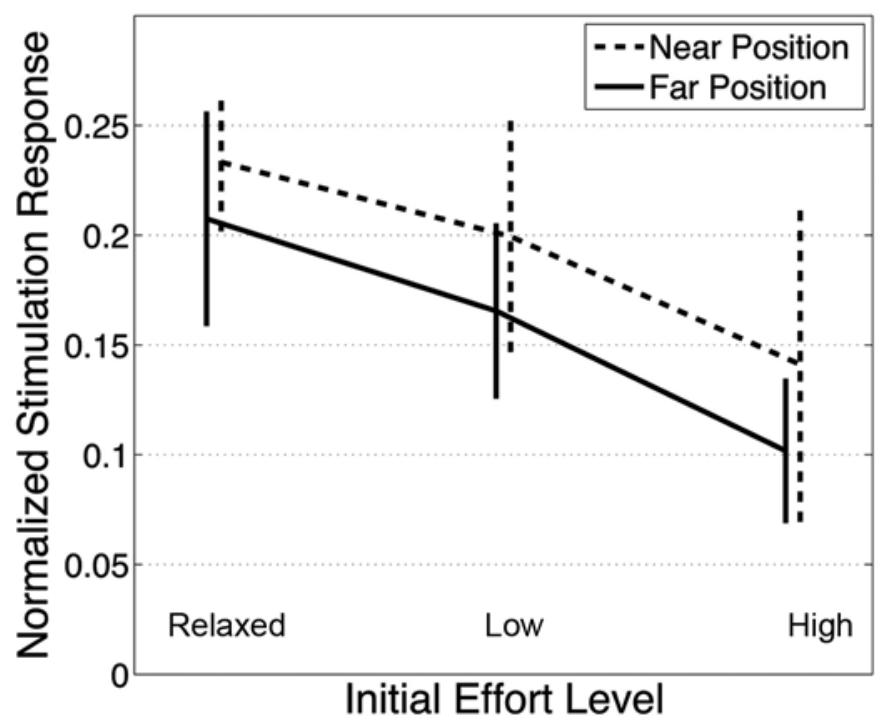

Figure 5.

Averaged stimulation response and standard deviation across participants.

the two effort levels. Table 3 shows that in 7 of the 10 instances, the increment in force during stimulation alone was significantly greater than during the combination of low effort and stimulation $(p<0.05)$ with a mean difference between the force increments for the two tasks of -18.0 percent. The force increment in six of those seven cases was still significantly greater when we subtracted the change in force during the voluntary effort maintenance task. At moderate (50\%) effort, eight of the stimulation-alone force increments were significantly greater than combined effort and stimulation force increments $(p<$ 0.05 ) with a mean difference of -44.9 percent. All eight of the force increments were still significantly greater when we subtracted the change in force from the voluntary effort maintenance from the combination trial.

The dependence of the normalized force increment on the voluntary effort maintenance task is described well by the equation $(\mathrm{Ft}-\mathrm{Fv}) / \mathrm{Fs}=\left(1+a^{*} \mathrm{Fvn}\right)$, where $\mathrm{Ft}=$ total force after onset of stimulation, $\mathrm{Fv}=$ voluntary force prior to onset of stimulation, Fs = force due to stimulation alone, Fvn = normalized voluntary force (magnitude of the voluntary force normalized by the vFmax $[\mathrm{Fvn}=$ $\mathrm{Fv} / \mathrm{vFmax}]$ ), and $a=$ slope parameter characterizing how force increment changes with voluntary effort. This equation is similar to the model used by Perumal et al. [17], except with the assumption that the change in force is linear over the range measured. We fit the equation to each participant's data separately, forcing the line through 1 on the vertical axis. The average value for the slope is -0.23 (standard deviation $=0.13$ ). The mean difference in slope between the near and far positions was 0.04 , but a paired $t$-test did not indicate statistical significance $(p=0.63)$.

While Figures 4(b), 4(d), and 5 and Table 3 show a decrease in the response to stimulation as voluntary effort increases, in all instances the stimulation response was statistically greater than zero $(p<0.05)$, indicating that stimulation increased the total force in all cases.

The target force directions among the set of participants covered a range of directions (Table 2). Across the participants, the target force directions followed a trend, with the more medial preferred directions ( $+z$ rotations) having smaller downward components ( $+y$ rotations). The directional rotations are with respect to the coordinate frame (Figure 1(a)). None of the participants had a target force direction in the same direction as the stimulated triceps force.

Table 3.

Summarized results showing number of instances where change in force in combined voluntary effort and stimulation task is different than change in force during stimulation alone, as well as number of instances when difference between those changes is different than change in maintained voluntary force during voluntary effort alone without either stimulation or feedback.

\begin{tabular}{|c|c|c|c|c|c|}
\hline $\begin{array}{c}\text { Effort } \\
\text { Combination }\end{array}$ & $\begin{array}{c}\text { Change } \\
\text { Significantly } \\
\text { Different From } \\
\text { Stimulation Alone }\end{array}$ & $\begin{array}{c}\text { Combination } \\
\text { Change as } \\
\text { Proportion of } \\
\text { Stimulation Alone } \\
(\%)\end{array}$ & $\begin{array}{c}\text { Mean Combination } \\
\text { Change Difference } \\
\text { (\%) }\end{array}$ & $\begin{array}{c}\text { Mean } \\
\text { Voluntary Change } \\
\text { (no stimulation) } \\
(\%)\end{array}$ & $\begin{array}{c}\text { Combination } \\
\text { Increment Minus } \\
\text { Voluntary } \\
\text { Maintenance } \\
\text { Significantly Different } \\
\text { from Stimulation } \\
\text { Alone }^{*}\end{array}$ \\
\hline Low & $7 / 10$ & 82.0 & -18.0 & -2.8 & $6 / 10$ \\
\hline Moderate & $8 / 10$ & 55.1 & -44.9 & -9.0 & $8 / 10$ \\
\hline
\end{tabular}


While the data set is incomplete for calculating the full set of inverse torque calculations, we can make some general observations about the directions of forces being generated by the elbow and shoulder based on the directions of end-point forces. The average angle between forces generated by the shoulder and elbow in the near and far positions were $62^{\circ}$ and $94^{\circ}$, respectively.

\section{DISCUSSION}

The results support hypothesis 1: FES can produce greater forward force even in the presence of some levels of voluntary effort. This increase in force should lead to increased movement produced with a neuroprosthesis. However, the results also support hypothesis 2: the force response depends on the effort level of the participant. The results also indicate that there may be individual variations in the relative dependence on voluntary force. On average, the force increment caused by stimulation was reduced by 15 percent in the presence of 20 percent voluntary effort and by 36 percent in the presence of 50 percent voluntary effort. While the results indicate that the total force is not a simple summation of voluntary and stimulated forces, the differences between individuals imply that a more targeted set of experiments to specifically determine the mechanisms that result in these changes and what is causing the variations across individuals would be useful.

These results are similar to those reported in nondisabled participants. Langzam et al. studied the tibialis anterior and observed that higher stimulation levels were necessary to produce the same total torque levels when superimposed on higher volitional torques [24]. They based their experimental and analysis methods on an explicit assumption that the voluntary and stimulated motor neurons did not overlap, as well as the quantitative estimation of voluntary contraction levels from the EMG of the stimulated tibialis anterior, whereas our experimental and analysis methods did not. Perumal et al. observed that stimulation superimposed on voluntary effort decreased the stimulation response with increasing voluntary effort in the quadriceps [17]. They assumed that motor unit recruitment overlap was the only mechanism contributing to the change in force. We studied a different muscle group in an impaired group of participants and also observed significant variations between participants and similar decreases in force increment.
Both the current study and the earlier studies observed less than linear summation of the stimulated and voluntary force responses that was force dependent. The similarity of the conclusions in the perspective of the differences in approach gives increased confidence in the robustness of the effect.

These experiments did not examine the mechanism creating these changes, but there are a few that could be causing this difference. One possible mechanism contributing to the reduction in incremental force is that some motor units are activated both by voluntary effort and by FES (overlap). If the motor units being recruited by the stimulation overlap with the volitionally activated motor units in the same muscle, the increment in force from stimulation will be limited to the additional recruitment of inactive motor units and the possible increase in excitation frequency of volitionally activated motor units. Even though the brain recruits in order of smallest to largest motor units [25] and it is generally accepted that neuromuscular electrical stimulation has been shown to recruit motor units in the opposite order using direct nerve stimulation [26], the response to surface stimulation has also been observed to be more mixed and unselective [26-27]. Despite the differences in recruitment order, rate modulation continues after full recruitment, with the result that full voluntary recruitment can occur well before maximal force is achieved [28-30]. This interpretation is consistent with the observation (Figure 4(b), (d)) that the reduction in the incremental force is larger at higher effort levels, since we would expect both greater overlap of recruitment and higher motor unit firing rates at the higher force level. Fatigue tests employing maximal voluntary activation have demonstrated that short trains of supramaximal stimulation do not produce a force increment [31]. Thus, we would not expect an increase in force if either stimulation or voluntary effort alone was fully activating the muscle. If the primary mechanism for the decrease in force increment is motor unit activation overlap, variations in the extent of overlap between the directions of the volitional force and stimulated force, including a lack of overlap in the near position for participant 3 , could partially explain the variations in force dependence across participants. In addition, participant 3's preferred voluntary force direction in the near position was the most different from the stimulated direction of all of the combinations of participants and positions. It is possible that overlap was low because the shoulder was contributing significantly to the 
force output and the elbow was not contributing much extension.

Another possible mechanism is that stimulation elicits reflexes that are modulated by effort. Surface stimulation would be expected to excite both cutaneous and proprioceptive afferents, and widespread reflexes in the arm following stroke are augmented by effort [23,32-33]. Reflexes could either enhance or reduce the total force. Since the changes in force increment are not significantly different $(p<0.05)$ between the near and far arm positions based on the slope of the model, this indicates that a position-dependent reflex effect is not significantly contributing to the difference in force increments. It should be noted, however, that this study is not powered to account for $\beta$ error, so while we cannot say that the force increments for the two positions are significantly different, we also cannot confidently say that they are the same.

Another explanation for the force increment caused by stimulation decreasing with increasing effort is a failure to maintain voluntary effort in the presence of both stimulation and a loss of feedback. However, our analysis indicates that failure to maintain effort because of loss of feedback is unlikely to be the primary explanation. While there was a decrease in the voluntary force generated when feedback was removed, the difference between the changes during stimulation alone and combined voluntary effort and stimulation was significantly greater than the change in force when feedback was removed during voluntary effort alone. This supports the hypothesis that the changes are a result of more than just the feedback removal during the task. There could also be a change in voluntary effort as a result of participants feeling and responding to the sensation of FES. Participants complete multiple training sessions before the test sessions to allow them to become comfortable with the sensation of FES; however, the current study cannot statistically verify that sensation is not a mechanism for the decrease in force increment.

The variance in combined force reduction across participants could partially be a reflection of the variance in synergy pattern expression. The maximum net force is partially limited by antagonist muscle cocontraction in the synergy patterns and participants potentially being unable to generate maximal contractions poststroke. Both of these would prevent knowing the maximum forces produced by individual muscles. The target forces and stimulated forces are scaled to the maximum net force, not the actual maximum individual muscle force. Thus, variation of the intensity of synergies across participants could lead to variation of effects. Similarly, if motor unit overlap is the mechanism responsible for the reduction, variation in recruitment and rate modulation patterns across participants [34-36] could lead to variability in the amount of voluntary effort that is replaced by FES.

The decrease in stimulation response with increasing voluntary effort is statistically significant for effort and the interaction between participants and effort, with a weaker dependence on the interaction. The sum of the squares of the effort is 0.41 , compared with 0.11 for the interaction between effort and participant, indicating that effort is generally the stronger contributor. In future studies, percutaneous stimulation, measurement of EMG levels across muscles, and an experimental design that simplifies force analysis could provide insight into the mechanisms responsible.

The experimental design could be improved by standardizing the arm position and orientation by joint angle rather than end-point position. This would allow choosing a consistent arm orientation across participants, making it easier to back-calculate joint torques. We cannot rule out contributions of shoulder torques to the endpoint force. Calculating the joint torques would enable assessments of the force contributions of different joints, similar to the method used by Keller et al. [9]. The current experimental design could not distinguish between forces generated by shoulder and elbow torques, as previously mentioned. Inverse joint torque calculations are sensitive to small changes in limb configuration, and we did not measure the horizontal arm orientation with adequate precision. As described in the "Setup" section, the small forces transmitted through the arm support would also affect the calculated joint torques, but the effect of these forces in our experimental setup is less than the effect of errors in the shoulder and elbow positions. Similarly, it would be ideal to have the same voluntary force target direction for each participant. Targeting joints that have fewer muscles crossing them would further simplify the interpretation of the data. Lastly, nondisabled trials incorporating these design changes would help establish a baseline before evaluating these summations in groups who have altered reflexes and central inputs. These changes would provide greater understanding of the mechanisms, which is important to exploit voluntary force augmentation in the future design of neuroprostheses.

The relationship between the response to stimulation and the underlying level of voluntary effort has potential 
ramifications for the design of stroke neuroprostheses that integrate a user's voluntary effort with FES. While there is a decrease in the force increment as voluntary effort increases, the force increment is still substantial $(64 \%)$ at moderate effort for a stimulation level that produces 20 percent vFmax when exerting no effort. Partial effort does not completely block the stimulation response, indicating that voluntary effort can be used as a command signal and FES can still augment movements as long as effort level is considered while designing poststroke neuroprosthesis control schemes. Limiting effort to limit the expression of synergy patterns will allow FES to have a greater effect. This type of neuroprosthesis would use EMG recorded at low-effort levels as the command signal for large levels of stimulation that would be the primary movement generators. The user's focus would be to generate suitable EMG levels for a command signal rather than attempting to generate maximum effort. As observed in the results of Keller et al. [9], shoulder abduction generates elbow flexion that can be difficult to overcome by elbow extensor stimulation. Our preliminary studies support the hypothesis that reducing voluntary effort during reach and hand opening and supplementing that effort with stimulation can generate hand opening even during reach [37]. By generating most of the shoulder abduction with stimulation instead of voluntary effort, elbow and hand stimulation may be able to have a greater net effect.

One approach in designing neuroprostheses for stroke is to rely as much as possible on the user's residual voluntary ability to move and only add stimulation to supplement the voluntary effort as needed. This approach minimizes the extent of intervention (number of channels, intensity of stimulation) and maximizes the potential therapeutic benefit of requiring greater voluntary control. However, there are three potential benefits to reducing voluntary effort and increasing the use of stimulation in a neural prosthesis. First, lower voluntary effort reduces the intensity of synergistic contraction patterns [6]. Synergy patterns scale somewhat proportionally to effort, increasing the forces in multiple muscles [5]. In order to decrease the undesired synergy response and maximize the stimulation response, reducing the effort exerted on the part of the user is beneficial. Thus, less stimulation force would be required to overcome undesired contractions of antagonists. For example, relaxation of the arm allows FES to open the hand by reducing synergistic finger flexor activation. While FES has been hypothesized to reduce antagonist contractions by reciprocal inhibition, experimental evidence does not support that hypothesis [15]. Second, there is no loss of maximal force potential by limiting voluntary effort because lower voluntary effort increases the forces that can be elicited by FES in the same muscle. Maximal stimulation and maximal voluntary activation produce the same force [31]. Third, lower effort reduces the intensity of stretch reflexes, which slow down and limit the extent of voluntary movement in stroke $[23,33]$.

If the mechanism of reduction in the stimulation response as a result of increased effort is a result of overlap between activated motor units, this has another implication for the design of neuroprostheses for stroke. For systems that use EMG from stimulated muscles as part of the control signal [38], one must be aware that some of the asynchronous action potentials activated by the central nervous system will be replaced by synchronous action potentials activated by stimulation. Even if the synchronous action potentials (M-waves) are removed from the signal [38-41], the residual EMG is not entirely indicative of the user's effort in that muscle.

FES, as a functional neuroprosthesis, has the potential to increase function by improving reach and hand opening [37], augmenting the gains in function that are achieved by physical rehabilitation and robotic therapies. Neuroprosthetic and therapeutic approaches are not mutually exclusive approaches to increasing either reach [20] or hand function [42] after stroke, and combined approaches may provide the best results. Assistive forces from an FES system may enable people with stroke to utilize some of these therapies that they were previously unable to participate in. Similarly, participating in these therapies may increase volitional movement and disconnect synergy patterns [20], thereby allowing more assistance from volitional effort, providing a more robust command signal, allowing for finer movements in response to stimulation, and progressively decreasing reliance on the stimulation.

Future stroke neuroprostheses should be designed with an understanding of the relationship between effort, stimulation level, and motor output. Doing so can allow the user to derive optimal benefit from the device. These results indicate that even in the presence of moderate voluntary effort, FES can increase poststroke force production, decreasing the impairment on the affected side. 


\section{CONCLUSIONS}

FES is capable of increasing end-point force even in the presence of voluntary effort poststroke. The stimulation response depends on the level of voluntary effort and to a lesser extent on the individual participant; however, the contributions of different mechanisms are unknown. The change in force response should be taken into consideration in the design of future poststroke neuroprostheses.

\section{ACKNOWLEDGMENTS}

\author{
Author Contributions: \\ Study concept and design: N. Makowski, J. Knutson, J. Chae, \\ P. Crago. \\ Acquisition of data: N. Makowski. \\ Analysis and interpretation of data: N. Makowski, P. Crago. \\ Drafting of manuscript: N. Makowski, P. Crago. \\ Critical revision of manuscript for important intellectual content: \\ N. Makowski, J. Knutson, J. Chae, P. Crago. \\ Statistical analysis: N. Makowski. \\ Obtained funding: J. Knutson, J. Chae, P. Crago.
}

Financial Disclosures: The authors have declared that no competing interests exist.

Funding/Support: This material is based on work supported by the National Institutes of Health National Institute of Child Health and Human Development (grant R21HD05256) and the National Institute of Biomedical Imaging and Bioengineering (grant T32-EB04314).

Additional Contributions: The authors would like to thank Maureen Hennessy, PT, and Terri Hisel, OT, for performing the impairment assessments, as well as Margaret Maloney, RN, for scheduling the study visits and Steven Sidik, $\mathrm{PhD}$, for providing consultation about the statistics.

Institutional Review: All participants provided informed consent in accordance with the Declaration of Helsinki prior to participation in this study, which was approved by an institutional review board. Participant Follow-up: The authors do not plan to inform participants of the publication of this study because it is not evaluating an immediate intervention or therapy and the participants themselves would likely not be interested in the results.

\section{REFERENCES}

1. Roger VL, Go AS, Lloyd-Jones DM, Adams RJ, Berry JD, Brown TM, Carnethon MR, Dai S, de Simone G, Ford ES, Fox CS, Fullerton HJ, Gillespie C, Greenlund KJ, Hailpern SM, Heit JA, Ho PM, Howard VJ, Kissela BM, Kittner SJ, Lackland DT, Lichtman JH, Lisabeth LD, Makuc DM, Marcus GM, Marelli A, Matchar DB, McDermott MM, Meigs JB, Moy CS, Mozaffarian D, Mussolino ME, Nichol G, Paynter NP, Rosamond WD, Sorlie PD, Stafford RS,
Turan TN, Turner MB, Wong ND, Wylie-Rosett J; American Heart Association Statistics Committee and Stroke Statistics Subcommittee. Heart disease and stroke statistics2011 update: a report from the American Heart Association. Circulation. 2011;123(4):e18-e209.

[PMID:21160056]

http://dx.doi.org/10.1161/CIR.0b013e3182009701

2. Twitchell TE. Sensory factors in purposive movement. J Neurophysiol. 1954;17(3):239-52. [PMID:13163711]

3. Dewald JP, Beer RF. Abnormal joint torque patterns in the paretic upper limb of subjects with hemiparesis. Muscle Nerve. 2001;24(2):273-83. [PMID:11180211]

4. Neckel N, Pelliccio M, Nichols D, Hidler J. Quantification of functional weakness and abnormal synergy patterns in the lower limb of individuals with chronic stroke. J Neuroeng Rehabil. 2006;3:17. [PMID:16857059] http://dx.doi.org/10.1186/1743-0003-3-17

5. Sukal TM, Ellis MD, Dewald JP. Shoulder abductioninduced reductions in reaching work area following hemiparetic stroke: neuroscientific implications. Exp Brain Res. 2007;183(2):215-23. [PMID:17634933] http://dx.doi.org/10.1007/s00221-007-1029-6

6. Beer RF, Ellis MD, Holubar BG, Dewald JP. Impact of gravity loading on post-stroke reaching and its relationship to weakness. Muscle Nerve. 2007;36(2):242-50. [PMID:17486581] http://dx.doi.org/10.1002/mus.20817

7. Kilgore KL, Peckham P, Keith MW. Twenty year experience with implanted neuroprostheses. Conf Proc IEEE Eng Med Biol Soc. 2009;2009:7212-15. [PMID:19965280] http://dx.doi.org/10.1109/IEMBS.2009.5335272

8. Giuffrida JP, Crago PE. Functional restoration of elbow extension after spinal-cord injury using a neural networkbased synergistic FES controller. IEEE Trans Neural Syst Rehabil Eng. 2005;13(2):147-52. [PMID:16003892] http://dx.doi.org/10.1109/TNSRE.2005.847375

9. Keller T, Ellis MD, Dewald JP. Overcoming abnormal joint torque patterns in paretic upper extremities using triceps stimulation. Artif Organs. 2005;29(3):229-32.

[PMID:15725223] http://dx.doi.org/10.1111/j.1525-1594.2005.29041.x

10. Chae J, Hart R. Intramuscular hand neuroprosthesis for chronic stroke survivors. Neurorehabil Neural Repair. 2003; 17(2):109-17. [PMID:12814056] http://dx.doi.org/10.1177/0888439003017002005

11. Alon G, McBride K, Ring H. Improving selected hand functions using a noninvasive neuroprosthesis in persons with chronic stroke. J Stroke Cerebrovasc Dis. 2002;11(2): 99-106. [PMID:17903863] http://dx.doi.org/10.1053/jscd.2002.127107

12. Ring H, Rosenthal N. Controlled study of neuroprosthetic functional electrical stimulation in sub-acute post-stroke 
rehabilitation. J Rehabil Med. 2005;37(1):32-36.

[PMID:15788330]

http://dx.doi.org/10.1080/16501970410035387

13. Chiou YH, Luh JJ, Chen SC, Chen YL, Lai JS, Kuo TS. Patient-driven loop control for hand function restoration in a non-invasive functional electrical stimulation system. Disabil Rehabil. 2008;30(19):1499-1505.

[PMID:19230219]

http://dx.doi.org/10.1080/09638280701615246

14. Popovic MR, Popovic DB, Keller T. Neuroprostheses for grasping. Neurol Res. 2002;24(5):443-52.

[PMID:12117312]

http://dx.doi.org/10.1179/016164102101200311

15. Hines AE, Crago PE, Billian C. Functional electrical stimulation for the reduction of spasticity in the hemiplegic hand. Biomed Sci Instrum. 1993;29:259-66. [PMID:8329599]

16. Kamper DG, Rymer WZ. Impairment of voluntary control of finger motion following stroke: role of inappropriate muscle coactivation. Muscle Nerve. 2001;24(5):673-81. [PMID:11317278] http://dx.doi.org/10.1002/mus.1054

17. Perumal R, Wexler AS, Kesar TM, Jancosko A, Laufer Y, Binder-Macleod SA. A phenomenological model that predicts forces generated when electrical stimulation is superimposed on submaximal volitional contractions. J Appl Physiol. 2010;108(6):1595-1604. [PMID:20299613] http://dx.doi.org/10.1152/japplphysiol.01231.2009

18. Fugl-Meyer AR, Jääskö L, Leyman I, Olsson S, Steglind S. The post-stroke hemiplegic patient. 1. a method for evaluation of physical performance. Scand J Rehabil Med. 1975; 7(1):13-31. [PMID:1135616]

19. Ellis MD, Acosta AM, Yao J, Dewald JP. Positiondependent torque coupling and associated muscle activation in the hemiparetic upper extremity. Exp Brain Res. 2007;176(4):594-602. [PMID:16924488] http://dx.doi.org/10.1007/s00221-006-0637-x

20. Ellis MD, Sukal-Moulton T, Dewald JP. Progressive shoulder abduction loading is a crucial element of arm rehabilitation in chronic stroke. Neurorehabil Neural Repair. 2009;23(8):862-69. [PMID:19454622]

http://dx.doi.org/10.1177/1545968309332927

21. Crago PE, Houk JC, Hasan Z. Regulatory actions of human stretch reflex. J Neurophysiol. 1976;39(5):925-35. [PMID:978238]

22. Burgess PR, Cooper TA, Gottlieb GL, Latash ML. The sense of effort and two models of single-joint motor control. Somatosens Mot Res. 1995;12(3-4):343-58.

[PMID:8834307]

http://dx.doi.org/10.3109/08990229509093667

23. Trumbower RD, Ravichandran VJ, Krutky MA, Perreault EJ. Contributions of altered stretch reflex coordination to arm impairments following stroke. J Neurophysiol. 2010; 104(6):3612-24. [PMID:20962072]

http://dx.doi.org/10.1152/jn.00804.2009

24. Langzam E, Nemirovsky Y, Isakov E, Mizrahi J. Muscle enhancement using closed-loop electrical stimulation: volitional versus induced torque. J Electromyogr Kinesiol. 2007;17(3):275-84. [PMID:16690326] http://dx.doi.org/10.1016/j.jelekin.2006.03.001

25. Gordon T, Thomas CK, Munson JB, Stein RB. The resilience of the size principle in the organization of motor unit properties in normal and reinnervated adult skeletal muscles. Can J Physiol Pharmacol. 2004;82(8-9):645-61. [PMID:15523522] http://dx.doi.org/10.1139/y04-081

26. Merletti R, Knaflitz M, DeLuca CJ. Electrically evoked myoelectric signals. Crit Rev Biomed Eng. 1992;19(4): 293-340. [PMID:1563271]

27. Gregory CM, Bickel CS. Recruitment patterns in human skeletal muscle during electrical stimulation. Phys Ther. 2005;85(4):358-64. [PMID:15794706]

28. Milner-Brown HS, Stein RB, Yemm R. The orderly recruitment of human motor units during voluntary isometric contractions. J Physiol. 1973;230(2):359-70.

[PMID:4350770]

29. Kukulka CG, Clamann HP. Comparison of the recruitment and discharge properties of motor units in human brachial biceps and adductor pollicis during isometric contractions. Brain Res. 1981;219(1):45-55. [PMID:7260629] http://dx.doi.org/10.1016/0006-8993(81)90266-3

30. De Luca CJ, Hostage EC. Relationship between firing rate and recruitment threshold of motoneurons in voluntary isometric contractions. J Neurophysiol. 2010;104(2):1034-46. [PMID:20554838] http://dx.doi.org/10.1152/jn.01018.2009

31. Bigland-Ritchie B. EMG and fatigue of human voluntary and stimulated contractions. Ciba Found Symp. 1981;82: 130-56. [PMID:6913468]

32. Musampa NK, Mathieu PA, Levin MF. Relationship between stretch reflex thresholds and voluntary arm muscle activation in patients with spasticity. Exp Brain Res. 2007; 181(4):579-93. [PMID:17476486] http://dx.doi.org/10.1007/s00221-007-0956-6

33. Sangani SG, Starsky AJ, McGuire JR, Schmit BD. Multijoint reflex responses to constant-velocity volitional movements of the stroke elbow. J Neurophysiol. 2009;102(3): 1398-1410. [PMID:19553478] http://dx.doi.org/10.1152/jn.90972.2008

34. Masakado Y, Noda Y, Nagata MA, Kimura A, Chino N, Akaboshi K. Macro-EMG and motor unit recruitment threshold: differences between the young and the aged. Neurosci Lett. 1994;179(1-2):1-4. [PMID:7845601] http://dx.doi.org/10.1016/0304-3940(94)90920-2 
35. Fling BW, Knight CA, Kamen G. Relationships between motor unit size and recruitment threshold in older adults: implications for size principle. Exp Brain Res. 2009; 197(2):125-33. [PMID:19565231]

http://dx.doi.org/10.1007/s00221-009-1898-y

36. Feiereisen P, Duchateau J, Hainaut K. Motor unit recruitment order during voluntary and electrically induced contractions in the tibialis anterior. Exp Brain Res. 1997; 114(1):117-23. [PMID:9125456] http://dx.doi.org/10.1007/PL00005610

37. Makowski NS, Knutson JS, Chae J, Crago P. Neuromuscular electrical stimulation to augment reach and hand opening after stroke. Conf Proc IEEE Eng Med Biol Soc. 2011;2011:3055-58. [PMID:22254984] http://dx.doi.org/10.1109/IEMBS.2011.6090835

38. Yeom H, Chang YH. Autogenic EMG-controlled functional electrical stimulation for ankle dorsiflexion control. J Neurosci Methods. 2010;193(1):118-25. [PMID:20713086] http://dx.doi.org/10.1016/j.jneumeth.2010.08.011

39. Frigo C, Ferrarin M, Frasson W, Pavan E, Thorsen R. EMG signals detection and processing for on-line control of functional electrical stimulation. J Electromyogr Kinesiol. 2000;10(5):351-60. [PMID:11018444] http://dx.doi.org/10.1016/S1050-6411(00)00026-2

40. Langzam E, Isakov E, Mizrahi J. Evaluation of methods for extraction of the volitional EMG in dynamic hybrid muscle activation. J Neuroeng Rehabil. 2006;3:27. [PMID:17123447] http://dx.doi.org/10.1186/1743-0003-3-27
41. Sennels S, Biering-Sørensen F, Andersen OT, Hansen SD. Functional neuromuscular stimulation controlled by surface electromyographic signals produced by volitional activation of the same muscle: adaptive removal of the muscle response from the recorded EMG-signal. IEEE Trans Rehabil Eng. 1997;5(2):195-206. [PMID:9184905] http://dx.doi.org/10.1109/86.593293

42. Wolf SL, Winstein CJ, Miller JP, Taub E, Uswatte G, Morris D, Giuliani C, Light KE, Nichols-Larsen D; EXCITE Investigators. Effect of constraint-induced movement therapy on upper extremity function 3 to 9 months after stroke: the EXCITE randomized clinical trial. JAMA. 2006;296(17): 2095-2104. [PMID:17077374]

http://dx.doi.org/10.1001/jama.296.17.2095

Submitted for publication April 7, 2011. Accepted in revised form May 23, 2012.

This article and any supplementary material should be cited as follows:

Makowski N, Knutson J, Chae J, Crago P. Interaction of poststroke voluntary effort and functional neuromuscular electrical stimulation. J Rehabil Res Dev. 2013;50(1): 85-98.

http://dx.doi.org/10.1682/JRRD.2011.04.0068

ResearcherID: Jayme Knutson, PhD: A-3607-2013

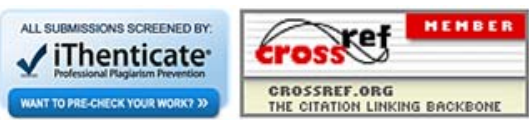

